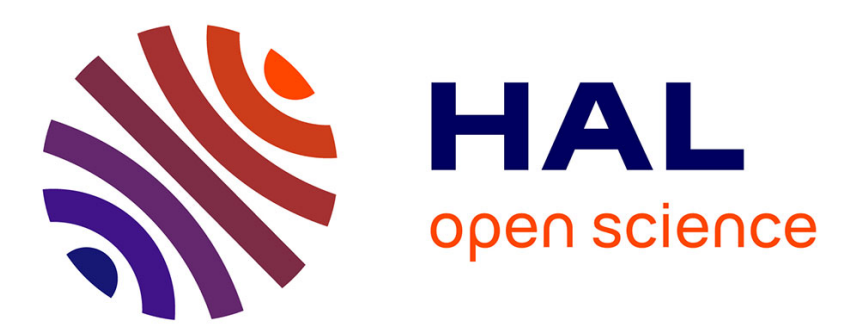

\title{
Proportional Transaction Costs in the Robust Control Approach to Option Pricing: The Uniqueness Theorem
}

Naïma El Farouq, Pierre Bernhard

\section{To cite this version:}

Naïma El Farouq, Pierre Bernhard. Proportional Transaction Costs in the Robust Control Approach to Option Pricing: The Uniqueness Theorem. Applied Mathematics and Optimization, 2015, 72 (2), pp.187-202. 10.1007/s00245-014-9276-y . hal-01090616

\section{HAL Id: hal-01090616 https://inria.hal.science/hal-01090616}

Submitted on 3 Dec 2014

HAL is a multi-disciplinary open access archive for the deposit and dissemination of scientific research documents, whether they are published or not. The documents may come from teaching and research institutions in France or abroad, or from public or private research centers.
L'archive ouverte pluridisciplinaire HAL, est destinée au dépôt et à la diffusion de documents scientifiques de niveau recherche, publiés ou non, émanant des établissements d'enseignement et de recherche français ou étrangers, des laboratoires publics ou privés. 


\title{
Proportional Transaction Costs in the Robust Control Approach to Option Pricing: the Uniqueness Theorem
}

\author{
Naïma El Farouq* $\quad$ Pierre Bernhard ${ }^{\dagger}$
}

September 17, 2014

\begin{abstract}
We prove the missing uniqueness theorem for the viscosity solution of a quasi-variational inequality related to a minimax impulse control problem modeling the option pricing with proportional transactions costs. This result makes our robust control approach of option pricing in the interval market model essentially complete.
\end{abstract}

Keywords Option pricing; viscosity solutions; robust control.

\section{Introduction}

In a series of papers $[4,5,8,9]$ culminating with [10], we have developed a robust control, probabilityfree approach of option pricing, for both vanilla and digital european options. We claim that, on the one hand, we have the possibility of constructing a consistent theory of hedging portfolios with either continuous or discrete time trading paradigms, with one and the same market model, and, on the other hand, we can accommodate transaction costs and closing costs in a natural way, with a nontrivial hedging portfolio.

We also argue that the probabilistic description of future securities prices, as used by the classic approaches, imbeds much more information on these prices future histories than does the interval market model of the robust control approach which only assumes bounds on the rate of relative variation. As a consequence, the new approach is less sensitive to modelling uncertainties.

The last missing item in our theory was a uniqueness theorem for the viscosity solution of a particular, highly degenerate, Isaacs quasi-variational inequality (QVI). This degeneracy comes first from the fact that the QVI is of first order, so it cannot enjoy the ellipticity property [3]. The other fact is that we assume that the transaction costs are proportional to the amount of transactions, so that the infimum of impulse costs is zero. We will show that the uniqueness of the viscosity solution of this QVI can not be guaranteed. We also recall the general result in [12] where uniqueness of the viscosity solution is proved under the assumption that the infimum of impulse costs is positive. That paper was motivated by our theory of option pricing where we consider that a fixed cost is charged for any transaction in addition to a variable part depending on the amount of the transaction.

\footnotetext{
${ }^{*}$ Université Blaise Pascal (Clermont-Ferrand II), Complexe scientifique des Cézeaux, 24 avenue des Landais, B.P. 80026, 63171 Aubière cedex, France. Naima.ElFarouq@univ-bpclermont.fr

${ }^{\dagger}$ INRIA Sophia Antipolis-Méditerranée, 2004 route des Lucioles, B.P. 93, 06902 Sophia Antipolis cedex, France. Pierre.Bernhard@inria.fr
} 
In [9], the authors replace the minimax impulse control problem with an equivalent problem without impulses. They prove that they have the same value function. In this paper, we deal with the associated differential QVI (DQVI). The technique in [11], where the author considers infinite-horizon time control problems where the infimum of impulse costs is zero is, in a large extent, different. The idea is to give a new QVI that admits the same viscosity subsolutions than the original one and to prove that the value function is the unique viscosity solution of the new QVI.

A first direct and different proof of uniqueness of the viscosity solution of our DQVI was presented in the conference paper [7].

In this article, we sketch the overall context and prove the uniqueness sought, with a new proof requiring less regularity on the data. Notice, however, that the present proof does not account for the discontinuous payment digital options, while the indirect argument of [6] can be extended to that case, thanks to the concept of barrier.

We are obliged to recall the basics of our modelling of the problem to introduce the DQVI. The uniqueness of its viscosity solution is the object of this article. A more detailed exposition can be found in [8], where the uniqueness theorem is not proved, and the more recent [10], where a complete theory of hedging in the interval market model is developed.

\section{Modeling}

\subsection{The robust control approach to option pricing}

In our previous papers, we have introduced and discussed a probability free, robust control approach to option pricing, as follows.

\subsubsection{Notation}

We use the following notation:

- $t \in[0, T]$ : time, $0=$ date when the option is sold, $T=$ exercise time.

- $u(t)$ : the time varying, unpredictable, market price of the underlying security.

- $v(t)$ : the currency value of the risky part of the hedging portfolio, invested in the underlying.

- $\tau(t)=\dot{u}(t) / u(t)$ : action of the market.

- $\tau^{-}<0$ and $\tau^{+}>0$ : fixed known parameters: lower and upper bounds of $\tau$.

- $\Psi=$ set of measurable functions from $[0, T]$ to $\left[\tau^{-}, \tau^{+}\right]$.

- $\xi(t)$ : control of the seller of the option, or "trader": the rate of buying (selling if negative) underlying stocks, assumed to be infinitely divisible and liquid.

- $t_{k}$ : control of the trader: time of $k$-th block buy or sale of underlying stock.

- $\xi_{k}$ : control of the trader: amount of the $k$-th block buy (if positive) or sale (if negative) of underlying stock.

- $\Xi$ : set of time distributions defined over $[0, T]$ which are the sum of a measurable function $\xi^{c}(\cdot)$ and a finite number of weighted translated Dirac impulses $\xi_{k} \delta\left(t-t_{k}\right)$. 
- $\varphi \in \Phi:$ strategy of the trader: $\varphi: \Psi \rightarrow \Xi: \tau(\cdot) \mapsto \xi(t)=\varphi(\tau(\cdot))(t)$ nonanticipative strategy including impulses that cause "jumps" $\xi_{k}$.

- $C^{+}>0$ and $C^{-}<0$ : fixed known parameters: the rates of the proportional transaction costs for buying resp. selling underlying stocks.

- $c^{+} \in\left[0, C^{+}\right]$and $c^{-} \in\left[C^{-}, 0\right]$ : fixed known parameters: same as $C^{+}$and $C^{-}$but for closing costs.

- $N(u, v)=\max \left\{c^{\varepsilon}(-v), u-K+c^{\varepsilon}(u-v)\right\}$ : terminal cost to the trader, sum of contractual commitment and closing costs. (See notation $c^{\varepsilon}(a)$ below.)

- $W(t, u, v)$ : Value function of the minimax problem to be solved, giving the premium sought as $P(u(0))=W(0, u(0), 0)$.

Further, for $Z \in\{\tau, C, c\}$ the notation $Z^{\varepsilon}$ stands for $\left(Z^{\varepsilon}, \varepsilon \in\{-,+\}\right)$, and for any $a \in \mathbb{R}, Z^{\varepsilon} a$ will mean $Z^{\operatorname{sign}(a)} a$. We also set

$$
\tau^{\star}=\max _{\varepsilon}\left|\tau^{\varepsilon}\right|, \quad C_{\star}=\min _{\varepsilon}\left|C^{\varepsilon}\right|
$$

\subsubsection{The minimax impulse control problem}

This section refers to the results of $[5,8,9]$ that we will need in the sequel. In these articles, we have shown that the premium sought can be obtained as the solution of the following minimax impulse control problem

\section{Dynamics}

$$
\begin{aligned}
\dot{u} & =\tau u, \\
\dot{v} & =\tau v+\xi^{c}, \\
v\left(t_{k}^{+}\right) & =v\left(t_{k}^{-}\right)+\xi_{k} .
\end{aligned}
$$

Equivalently, we may describe $v$ with equation (3) only, including the jumps (4) as impulses in $\xi(\cdot)$. Accordingly, from here on, we let $\xi \in \Xi$ be the sum of a measurable function $\xi^{c}(\cdot)$ and a finite number of weighted translated Dirac:

$$
\xi(t)=\xi^{c}(t)+\sum_{k} \xi_{k} \delta\left(t-t_{k}\right)
$$

Criterion The optimum (least safe) premium, or seller's price, is obtained as ${ }^{1}$

$$
P\left(u_{0}\right)=\inf _{\varphi \in \Phi} \sup _{\tau(\cdot) \in \Psi}\left[N(u(T), v(T))+\int_{0}^{T}\left(-\tau(t) v(t)+C^{\varepsilon} \xi(t)\right) \mathrm{d} t\right],
$$

where the time functions $u(\cdot)$ and $v(\cdot)$ are generated from $u(0)=u_{0}$ and $v(0)=0$ by equations (2),(3) and $\xi(\cdot)=\varphi(\tau(\cdot))$.

\footnotetext{
${ }^{1}$ According to the above, the integral term in $\xi$ includes the terms $\sum_{k} C^{\varepsilon_{k}} \xi_{k}$
} 


\subsection{Solving the minimax impulse control problem}

\subsubsection{The related QVI and DQVI}

In a classical fashion we introduce its Isaacs value function:

$$
W(t, u, v)=\inf _{\varphi \in \Phi} \sup _{\tau(\cdot) \in \Psi}\left[N(u(T), v(T))+\int_{t}^{T}\left(-\tau(s) v(s)+C^{\varepsilon} \xi(s)\right) \mathrm{d} s\right],
$$

where the dynamics are integrated from $u(t)=u, v(t)=v$. Hence the seller's price is $P\left(u_{0}\right)=$ $W\left(0, u_{0}, 0\right)$.

The natural QVI [3, pp 9-10] associated to our problem is

$$
\begin{aligned}
& 0=\max \left\{-\frac{\partial W}{\partial t}+\min _{\tau \in\left[\tau^{-}, \tau^{+}\right]} \sup _{\xi \in \mathbb{R}}\left[-\tau \frac{\partial W}{\partial u} u+\tau\left(1-\frac{\partial W}{\partial v}\right) v-\xi \frac{\partial W}{\partial v}-C^{\varepsilon} \xi\right],\right. \\
& \left.W(t, u, v)-\min _{\xi \in \mathbb{R}}\left[W(t, u, v+\xi)+C^{\varepsilon} \xi\right]\right\} \\
& \forall(u, v) \in \mathbb{R}^{2}, \quad W(T, u, v)=N(u, v) .
\end{aligned}
$$

Note that if $\frac{\partial W}{\partial v}+C^{-}>0$, then by choosing $\xi$ going to $-\infty$, then $-\xi \frac{\partial W}{\partial v}-C^{-} \xi$ will go to $+\infty$ and this is in contradiction with QVI (6). If $\frac{\partial W}{\partial v}+C^{+}<0$, then by choosing $\xi$ going to $+\infty$, then $-\xi \frac{\partial W}{\partial v}-C^{+} \xi$ will go to $+\infty$ and this is also in contradiction with QVI (6). We therefore necessarily have

$$
C^{-} \leq-\frac{\partial W}{\partial v} \leq C^{+}
$$

It is then easy to see that the $\sup _{\xi}$ in this QVI is obtained for $\xi=0$. So, QVI (6) is equivalent to (7) and the following new QVI

$$
\begin{gathered}
0=\max \left\{-\frac{\partial W}{\partial t}+\min _{\tau \in\left[\tau^{-}, \tau^{+}\right]} \tau\left[-\frac{\partial W}{\partial u} u+\left(1-\frac{\partial W}{\partial v}\right) v\right],\right. \\
\left.W(t, u, v)-\min _{\xi \in \mathbb{R}}\left[W(t, u, v+\xi)+C^{\varepsilon} \xi\right]\right\}, \\
\forall(u, v) \in \mathbb{R}^{2}, \quad W(T, u, v)=N(u, v) .
\end{gathered}
$$

There are new features in that game, in that, on the one hand, impulse controls are allowed, but, on the other hand, impulse costs have a zero infimum. It is easy to see that any function satisfies the viscosity supersolution inequality and that any constant function is a viscosity solution to this QVI, so we cannot prove that the value function of the impulse minimax problem is the unique viscosity solution of this QVI.

In [9], we introduced the so-called "Joshua transformation" that changes the minimax impulse control problem into an equivalent problem without impulse controls. We briefly explain here this transformation and the results obtained in that paper.

A new artificial time $s$ and a new trader's control $j \in\{-1,0,1\}$ are introduced. Natural time $t$ becomes a state variable that stops when $j \neq 0$. The jumps amplitudes $\left|\xi_{k}\right|$ are now the sizes of such 
$s$-intervals. Hence, jumps in $v$ have been turned into continuous variations in artificial time. When $j=0$, natural time and artificial time have equal variations.

By noting with a prime the derivatives with respect to $s$, and by $\bar{\jmath}=1-|j|$, the dynamics of this game are

$$
\left\{\begin{array}{l}
t^{\prime}=\overline{\mathrm{j}}, \\
u^{\prime}=\overline{\mathrm{j}} \tau u, \\
v^{\prime}=\overline{\mathrm{j}}(\tau v+\xi)+j,
\end{array}\right.
$$

In these dynamics, $\xi(s)$ contains no impulse anymore. Let $t(S)=T$. The new cost function is

$$
J(0, u(0), v(0), j(\cdot), \xi(\cdot), \tau(\cdot))=\int_{0}^{S}\left(\overline{\mathrm{j}}(s)\left(-\tau(s) v(s)+C^{\varepsilon} \xi(s)\right)+C^{\varepsilon} j\right) \mathrm{d} s+N(u(S), v(S)) .
$$

This differential game is stationary. Therefore, its Value function does not depend on $s$. In [9], we proved the relatively obvious fact that it is the same as the Value function of the original game:

$$
W(0, u(0), v(0))=\inf _{j(\cdot)} \inf _{\xi(\cdot)} \sup _{\tau(\cdot)} J(0, u(0), v(0), j(\cdot), \xi(\cdot), \tau(\cdot))
$$

The Isaac's equation of this new problem is the following:

$$
\begin{aligned}
& \forall(t, u, v) \in\left[0, T\left[\times \mathbb{R}^{2},\right.\right. \\
& 0=\max _{j \in\{-1,0,1\}}\left\{-\frac{\partial W}{\partial t} \overline{\mathrm{j}}+\min _{\tau \in\left[\tau^{-}, \tau^{+}\right]} \sup _{\xi \in \mathbb{R}}\left[-\overline{\mathrm{j}} \frac{\partial W}{\partial v} \xi-\overline{\mathrm{j}} C^{\varepsilon} \xi\right.\right. \\
& \left.\left.-\frac{\partial W}{\partial u} \overline{\mathrm{j}} \tau u-\frac{\partial W}{\partial v}(\overline{\mathrm{j}} \tau v+j)-C^{\varepsilon} j+\overline{\mathrm{j}} \tau v\right]\right\}, \\
& \forall(u, v) \in \mathbb{R}^{2}, \quad W(T, u, v)=N(u, v) .
\end{aligned}
$$

Successively replacing $j$ by either 0 (in this case with the same reasoning as above, the sup in $\xi$ is obtained for $\xi=0$,) 1 and -1 , this equation writes as the following "differential QVI" (DQVI):

$$
\begin{aligned}
& \forall(t, u, v) \in\left[0, T\left[\times \mathbb{R}^{2},\right.\right. \\
& 0=\max \left\{-\frac{\partial W}{\partial t}+\min _{\tau \in\left[\tau^{-}, \tau^{+}\right]} \tau\left[-\frac{\partial W}{\partial u} u+\left(1-\frac{\partial W}{\partial v}\right) v\right],\right. \\
& \left.-\left(\frac{\partial W}{\partial v}+C^{+}\right), \quad \frac{\partial W}{\partial v}+C^{-}\right\}, \\
& \forall(u, v) \in \mathbb{R}^{2}, \quad W(T, u, v)=N(u, v) .
\end{aligned}
$$

Applying standard results of Differential Games (see e.g. [14]) to the Isaacs equation associated to the Joshua-transformed problem, we easily get the following fact:

Theorem 2.1 The function $W$ defined by (5) is a continuous viscosity solution of DQVI (8).

But classic results such as $[1,2,13,14]$ do not apply here to prove the uniqueness of a viscosity solution. 


\subsubsection{Uniqueness}

In [8], we show that if the viscosity solution of the DQVI (8) can be proved unique, we have an interesting representation formula for the Value function of the continuous trading problem, a convergence theorem of the Value function $W^{h}$ of a discrete trading version of the problem to the Value function of the continuous trading problem as the step size $h$ vanishes, and a fast algorithm to compute the Value function of the discrete trading problem and the associated optimal trading strategy. Hence the importance of proving that uniqueness.

In order to exploit the technical result of the next section, we need to introduce a modified problem.

Let $R$ be a fixed positive number, and $\mathcal{R} \subset \mathbb{R}_{+} \times \mathbb{R}$ be the region $u \in[0, R],|v| \leq R$. For the time being, we consider only problems of option hedging where $(u(0), v(0)) \in \mathcal{R}$. As a consequence, for these problems, and for all $t \in[0, T]$, we have $u(t) \leq R \mathrm{e}^{\tau^{+} T}$.

Concerning $v(\cdot)$, the control $\xi$ might send it anywhere in $\mathbb{R}$. But we know from the analysis according to the Isaacs-Breakwell theory that the minimizing strategies never create large $v(t)$ 's. As a matter of fact, let $W_{0}$ be the maximum payoff obtained by the strategy $\varphi=0$ (after maximization in $\tau(\cdot))$ for any $(u(0), v(0))=\left(u_{0}, v_{0}\right) \in \mathcal{R}$. Let $a$ be a large number, chosen satisfying $a>$ $2 \exp \left(\tau^{+} T\right)\left[W_{0} /\left(R C_{\star}\right)+1\right]$, and $S=a R$. We claim the following:

Proposition 2.1 Any nonanticipative strategy $\varphi$ that causes $|v|>S$ is dominated by the strategy $\varphi=0$.

Proof Let $\zeta$ be a positive number, $\zeta<C_{\star} \exp \left(-\tau^{+} T\right) / 4 \tau^{+}$. Any nonanticipative strategy $\varphi$ may be challenged by the control function generated by the following rule: If $v(t-\zeta)>S$, choose $\tau(t)=\tau^{-}$if $v(t-\zeta)<-S$ choose $\tau(t)=\tau^{+}$. Due to the small time delay $\zeta$, it does generate an admissible control function $\tau(\cdot)$ against a nonanticipative strategy $\varphi$. Notice that, because $\varphi$ has to be non-anticipative, if a strategy $\varphi$ is to cause $|v|>S$, it also does so against that response of $\tau$, which comes after the fact.

It is easy to check that whether we reach $v=S$ or $v=-S$, from $\left|v_{0}\right| \leq R$, the cost $\int C^{\varepsilon} \xi(t) \mathrm{d} t$ is larger than $C_{\star}\left(\mathrm{e}^{-\tau^{+} T} S-R\right)$.

Consider, for instance, the case $v(t-\zeta) \geq S$, under the rule proposed to generate $\tau(\cdot)$, after a delay $\zeta$, we shall have either of three possibilities:

1. $v(t)>0$, thus $v(t) \tau(t)<0$,

2. $-S<v(t)<0$, we are back in the "small $v$ " domain, (and the minimizer has used twice the expense to produce large excursions in $v$ ),

3. $v(t) \leq-S$, we start a new sequence of the same analysis, with the opposite sign.

In the three cases, the benefit accrued to the minimizer from this large $|v|$ is not more than

$$
\int_{t-\zeta}^{t} S \mathrm{e}^{\tau^{+}(s-t+\zeta)} \mathrm{d} s=S\left(\exp \left(\tau^{+} \zeta\right)-1\right)<2 S \tau^{+} \zeta<C_{\star} \exp \left(-\tau^{+} T\right) S / 2 .
$$

Hence, any such excursion in $v$ costs the minimizer at least

$$
C_{\star}\left(\exp \left(-\tau^{+} T\right) a / 2-1\right) R>W_{0} .
$$

Since the terminal cost $N(u(T), v(T))$ is itself non negative, that strategy $\varphi$ does less well than $\varphi=0$. 
As a consequence, for initial states in $\mathcal{R}$, we may, without modifying the Value, restrict the set of admissible strategies to strategies that keep $|v| \leq S .^{2}$ With these strategies, the term $\int \tau(t) v(t) \mathrm{d} t$ is bounded. And therefore, as useful strategies $\varphi$ should keep $\int C^{\varepsilon} \xi(t) \mathrm{d} t-\int \tau(t) v(t) \leq W_{0}$, we can furthermore, without modifying the Value, restrict the admissible strategies to be such that $\int C^{\varepsilon} \xi(t) \mathrm{d} t$ be also bounded, and therefore also $\int|\xi(t)| \mathrm{d} t$. (Say, by $\left(W_{0}+1+\tau^{\star} S T\right) / C_{\star}$.)

Let $\Phi_{b}$ the set of admissible non anticipative strategies thus restricted.

We now modify the original problem as follows: let $\mathcal{P}_{[a, b]}$ be the projection of $\mathbb{R}$ on $[a, b] \subset \mathbb{R}$ and,

$$
N_{b}(u, v)=N\left(\mathcal{P}_{\left[0, \mathrm{e}^{\tau+T} R\right]}(u), \mathcal{P}_{[-S, S]}(v)\right), \quad L_{b}(v)=\mathcal{P}_{[-S, S]}(v) .
$$

We keep the same dynamics as we had, and define the pay-off as

$$
W_{b}\left(0, u_{0}, v_{0}\right)=\inf _{\varphi \in \Phi_{b}} \sup _{\tau \in \Psi}\left[N_{b}(u(T), v(T))+\int_{0}^{T}\left(-\tau L_{b}(v(t))+C^{\varepsilon} \xi(t)\right) \mathrm{d} t\right] .
$$

We have modified the problem only for states outside $\left[0, \mathrm{e}^{\tau^{+} T} R\right] \times[-S, S]$, never reached from initial states $\left(u_{0}, v_{0}\right)$ in $\mathcal{R}$. Hence in $\mathcal{R}$, the value of the modified game coincides with that of the original game:

$$
\left.W_{b}\right|_{\mathcal{R}}=\left.W\right|_{\mathcal{R}} .
$$

The next proposition follows from standard results (see e.g. $[13,14])$.

Proposition 2.2 The function $W_{b}$ is a bounded continuous viscosity solution of the modified DQVI:

$$
\begin{aligned}
& \forall(t, u, v) \in\left[0, T\left[\times \mathbb{R}^{2},\right.\right. \\
& \begin{aligned}
& 0=\max \left\{-\frac{\partial W_{b}}{\partial t}+\min _{\tau \in\left[\tau^{-}, \tau^{+}\right]} \tau\left[-\frac{\partial W_{b}}{\partial u} u-\frac{\partial W_{b}}{\partial v} v+L_{b}(v)\right],\right. \\
&\left.-\left(\frac{\partial W_{b}}{\partial v}+C^{+}\right), \quad \frac{\partial W_{b}}{\partial v}+C^{-}\right\}, \\
& \forall(u, v) \in \mathbb{R}^{2}, \quad W_{b}(T, u, v)=N_{b}(u, v) .
\end{aligned}
\end{aligned}
$$

We prove in the next section the following technical result.

Theorem 2.2 The DQVI (12) admits a unique bounded continuous viscosity solution.

Now, in view of the equality (11) and the fact that $\mathcal{R}$ could be chosen at will, all uniqueness results we need for the full theory follow.

\section{Proof of the uniqueness theorem}

We now set to prove theorem (2.2). We omit all indices $b$, but it should be understood all along that we are dealing with the modified problem.

\footnotetext{
${ }^{2}$ We may notice that from a modelling viewpoint, we might as well have introduced such a constraint a priori, from market liquidity considerations.
} 


\subsection{The Comparison Theorem}

\subsubsection{Modified DQVI's}

We first apply a classical transformation to DQVI (12) introducing

$$
\Gamma(t, u, v):=\mathrm{e}^{t} W_{b}(t, u, v),
$$

which is bounded if and only if $W_{b}$ is.

Proposition 3.1 The function $W_{b}$ is a viscosity solution of (12) if and only if $\Gamma$ is a viscosity solution of the modified DQVI:

$$
\begin{array}{r}
\forall(t, u, v) \in[0, T) \times \mathbb{R}^{2}, \\
0=\max \left\{-\frac{\partial \Gamma}{\partial t}+\Gamma(t, u, v)+\min _{\tau \in\left[\tau^{-}, \tau^{+}\right]} \tau\left[-\frac{\partial \Gamma}{\partial u} u-\frac{\partial \Gamma}{\partial v} v+\mathrm{e}^{t} L(v)\right],\right. \\
\left.-\frac{\partial \Gamma}{\partial v}-\mathrm{e}^{t} C^{+}, \frac{\partial \Gamma}{\partial v}+\mathrm{e}^{t} C^{-}\right\}, \\
\forall(u, v) \in \mathbb{R}^{2}, \quad \Gamma(T, u, v)=\mathrm{e}^{T} N(u, v) .
\end{array}
$$

Theorem 3.1 If $U$ is a bounded and upper semi-continuous viscosity subsolution of (13) and $V$ is a bounded lower semi-continuous viscosity supersolution of (13), then $U \leq V$ in $[0, T] \times \mathbb{R}^{2}$.

We need the following Lemma for the proof of this theorem. Let us recall the definition of the quantities $\tau^{*}$ and $C_{*}$ in equations (1).

Lemma 3.1 Let $U$ be an upper semi-continuous viscosity subsolution of (13), then for any $\mu \in(0,1)$, $\alpha>0$ and $K$ satisfying the following conditions,

$$
\begin{aligned}
\alpha & <2(1-\mu) C_{*}, \\
K & >2 C_{*} \tau^{*}+\mathrm{e}^{T} \tau^{*}\|L\|_{\infty},
\end{aligned}
$$

the function

$$
Z(t, u, v)=\mu U(t, u, v)-\frac{\alpha}{2} \ln \left(1+u^{2}\right)-\frac{\alpha}{2} \ln \left(1+v^{2}\right)-K(1-\mu)
$$

is a strict viscosity subsolution of (13).

Proof Observe first that $Z$ is an upper semi-continuous function. Let $\Phi \in C^{1}\left([0, T) \times \mathbb{R}^{2}\right)$ and $(\bar{t}, \bar{u}, \bar{v})$ a global maximum point of $Z-\Phi$.

We have that for all $(t, u, v) \in[0, T) \times \mathbb{R}^{2}$,

$$
\begin{aligned}
& U(\bar{t}, \bar{u}, \bar{v})-\frac{1}{\mu}\left(\frac{\alpha}{2} \ln \left(1+\bar{u}^{2}\right)+\frac{\alpha}{2} \ln \left(1+\bar{v}^{2}\right)+\Phi(\bar{t}, \bar{u}, \bar{v})\right) \geq \\
& U(t, u, v)-\frac{1}{\mu}\left(\frac{\alpha}{2} \ln \left(1+u^{2}\right)+\frac{\alpha}{2} \ln \left(1+v^{2}\right)+\Phi(t, u, v)\right) .
\end{aligned}
$$


This inequality means that $(\bar{t}, \bar{u}, \bar{v})$ is a global maximal point of $U-\Phi_{1}$ where

$$
\Phi_{1}(t, u, v)=\frac{1}{\mu}\left(\frac{\alpha}{2} \ln \left(1+u^{2}\right)+\frac{\alpha}{2} \ln \left(1+v^{2}\right)+\Phi(t, u, v)\right),
$$

then

$$
\begin{array}{r}
\text { at } \bar{t}, \bar{u}, \bar{v}, \quad \max \left\{-\frac{\partial \Phi_{1}}{\partial t}+U+\min _{\tau \in\left[\tau^{-}, \tau^{+}\right]} \tau\left[-\frac{\partial \Phi_{1}}{\partial u} \bar{u}-\frac{\partial \Phi_{1}}{\partial v} \bar{v}+\mathrm{e}^{\bar{t}} L(\bar{v})\right],\right. \\
\left.-\frac{\partial \Phi_{1}}{\partial v}-\mathrm{e}^{\bar{t}} C^{+}, \frac{\partial \Phi_{1}}{\partial v}+\mathrm{e}^{\bar{t}} C^{-}\right\} \leq 0
\end{array}
$$

that is

$$
\begin{aligned}
-\frac{\partial \Phi}{\partial t}+\mu U+\min _{\tau \in\left[\tau^{-}, \tau^{+}\right]} \tau & {\left[-\frac{\partial \Phi}{\partial u} \bar{u}-\frac{\alpha \bar{u}^{2}}{1+\bar{u}^{2}}-\frac{\partial \Phi}{\partial v} \bar{v}-\frac{\alpha \bar{v}^{2}}{1+\bar{v}^{2}}+\mu \mathrm{e}^{\bar{t}} L(\bar{v})\right] \leq 0, } \\
& -\frac{\partial \Phi}{\partial v}-\frac{\alpha \bar{v}}{1+\bar{v}^{2}}-\mu \mathrm{e}^{\bar{t}} C^{+} \leq 0, \\
& \frac{\partial \Phi}{\partial v}+\frac{\alpha \bar{v}}{1+\bar{v}^{2}}+\mu \mathrm{e}^{\bar{t}} C^{-} \leq 0 .
\end{aligned}
$$

Inequality (15) implies

$$
-\frac{\partial \Phi}{\partial v}-\mathrm{e}^{\bar{t}} C^{+} \leq(\mu-1) \mathrm{e}^{\bar{t}} C^{+}+\frac{\alpha \bar{v}}{1+\bar{v}^{2}} \leq(\mu-1) C^{+}+\frac{\alpha}{2} \leq(\mu-1) C_{*}+\frac{\alpha}{2} .
$$

Inequality (16) implies

$$
\frac{\partial \Phi}{\partial v}+\mathrm{e}^{\bar{t}} C^{-} \leq-\frac{\alpha \bar{v}}{1+\bar{v}^{2}}+(1-\mu) \mathrm{e}^{\bar{t}} C^{-} \leq(1-\mu) C^{-}+\frac{\alpha}{2} \leq(\mu-1) C_{*}+\frac{\alpha}{2} .
$$

Let $\delta=(\mu-1) C_{*}+\frac{\alpha}{2}$. Then, by choosing $0<\alpha<2(1-\mu) C_{*}$, we get $\delta<0$. Inequality (14) implies

$$
\begin{aligned}
& -\frac{\partial \Phi}{\partial t}+Z(\bar{t}, \bar{u}, \bar{v})+ \\
& \min _{\tau \in\left[\tau^{-}, \tau^{+}\right]} \tau\left[-\frac{\partial \Phi}{\partial u} \bar{u}-\frac{\alpha \bar{u}^{2}}{1+\bar{u}^{2}}-\frac{\partial \Phi}{\partial v} \bar{v}-\frac{\alpha \bar{v}^{2}}{1+\bar{v}^{2}}+\mu \mathrm{e}^{\bar{t}} L(\bar{v})\right] \leq K(\mu-1),
\end{aligned}
$$

then

$$
\begin{aligned}
& -\frac{\partial \Phi}{\partial t}+Z(\bar{t}, \bar{u}, \bar{v})+\min _{\tau \in\left[\tau^{-}, \tau^{+}\right]} \tau\left[-\frac{\partial \Phi}{\partial u} \bar{u}-\frac{\partial \Phi}{\partial v} \bar{v}+\mathrm{e}^{\bar{t}} L(\bar{v})\right] \leq \\
& -\frac{\partial \Phi}{\partial t}+Z(\bar{t}, \bar{u}, \bar{v}) \\
& \quad+\min _{\tau \in\left[\tau^{-}, \tau^{+}\right]} \tau\left[-\frac{\partial \Phi}{\partial u} \bar{u}-\frac{\alpha \bar{u}^{2}}{1+\bar{u}^{2}}-\frac{\partial \Phi}{\partial v} \bar{v}-\frac{\alpha \bar{v}^{2}}{1+\bar{v}^{2}}+\mu \mathrm{e}^{\bar{t}} L(\bar{v})\right] \\
& \quad+\max _{\tau \in\left[\tau^{-}, \tau^{+}\right]} \tau\left(\frac{\alpha \bar{u}^{2}}{1+\bar{u}^{2}}+\frac{\alpha \bar{v}^{2}}{1+\bar{v}^{2}}+(1-\mu) \mathrm{e}^{\bar{t}} L(\bar{v})\right) \leq \\
& K(\mu-1)+\max _{\tau \in\left[\tau^{-}, \tau^{+}\right]} \tau\left(\frac{\alpha \bar{u}^{2}}{1+\bar{u}^{2}}+\frac{\alpha \bar{v}^{2}}{1+\bar{v}^{2}}+(1-\mu) \mathrm{e}^{\bar{t}} L(\bar{v})\right) \leq \\
& 2 \alpha \tau^{*}+(\mu-1)\left(K-\mathrm{e}^{T} \tau^{*}\|L\|_{\infty}\right) \leq \\
& (\mu-1)\left(K-2 C_{*} \tau^{*}-\mathrm{e}^{T} \tau^{*}\|L\|_{\infty}\right) .
\end{aligned}
$$


Let $\delta^{\prime}=(\mu-1)\left(K-2 C_{*} \tau^{*}-\mathrm{e}^{T} \tau^{*}\|L\|_{\infty}\right)$. Choose $K>2 C_{*} \tau^{*}+\mathrm{e}^{T} \tau^{*}\|L\|_{\infty}$, to get $\delta^{\prime}<0$.

Now, let $V$ be a viscosity supersolution of (13), and let

$$
R=\exp \left(\frac{\|U\|_{\infty}+\|V\|_{\infty}}{\alpha}\right), \quad \mathcal{R}=[0, T] \times(-R, R) \times(-R, R),
$$

and for any function $X: \mathcal{R} \rightarrow \mathbb{R},\|X\|_{\mathcal{R}}=\sup _{t, u, v \in \mathcal{R}}|X(t, u, v)|$. Then, for all $(t, u, v) \notin \mathcal{R}$, we have $Z(t, u, v) \leq V(t, u, v)$. Indeed, for all $(t, u, v)$ such that $\max \{|u|,|v|\} \geq R$,

$$
Z(t, u, v) \leq(\mu-1)\|U\|_{\infty}-\|V\|_{\infty} \leq V(t, u, v) .
$$

It remains to prove this inequality for $(t, u, v) \in \mathcal{R}$.

Let

$$
\mathbb{M}=\sup _{(t, u, v) \in[0, T] \times \mathbb{R} \times \mathbb{R}}[Z(t, u, v)-V(t, u, v)] .
$$

If $\mathbb{M}$ is non-positive, then the proof is finished. If $\mathbb{M}$ is positive, then $\mathbb{M}=\sup _{(t, u, v) \in \mathcal{R}}(Z-V)$. Let us consider the test-function $\Phi_{\gamma}: \mathcal{R} \times \mathcal{R} \rightarrow \mathbb{R}$ :

$$
\Phi_{\gamma}\left(t, u, v, t^{\prime}, u^{\prime}, v^{\prime}\right)=Z(t, u, v)-V\left(t^{\prime}, u^{\prime}, v^{\prime}\right)-\frac{\left|u-u^{\prime}\right|^{2}}{\gamma^{2}}-\frac{\left|v-v^{\prime}\right|^{2}}{\gamma^{2}}-\frac{\left|t-t^{\prime}\right|^{2}}{\gamma^{2}} .
$$

Let $\left(\bar{t}, \bar{u}, \bar{v}, \bar{t}^{\prime}, \bar{u}^{\prime}, \bar{v}^{\prime}\right)$ be the maximum point of $\Phi_{\gamma}$. It exists since on the one hand, this is a bounded and upper semi-continuous function on a bounded set, and on the other hand, it is negative in a neighborhood of the boundary $|u|=R$ or $|v|=R$, and, by hypothesis, positive for some $(t, u, v)=$ $\left(t^{\prime}, u^{\prime}, v^{\prime}\right)$. Thus the search for the maximum may be restricted to a compact set $[0, T] \times[-R+\epsilon, R-$ $\epsilon]^{2}$. Let $\mathbb{M}_{\gamma}=\Phi_{\gamma}\left(\bar{t}, \bar{u}, \bar{v}, \bar{t}^{\prime}, \bar{u}^{\prime}, \bar{v}^{\prime}\right)$. We need the following lemma for the proof of Theorem 3.1.

Lemma 3.2 Assume that $\mathbb{M}>0$. Then, for any $\beta>0$, there exists $\gamma_{0}$ such that, for any $\gamma \leq \gamma_{0}$,

$$
\frac{\left|\bar{u}-\bar{u}^{\prime}\right|^{2}}{\gamma^{2}}+\frac{\left|\bar{v}-\bar{v}^{\prime}\right|^{2}}{\gamma^{2}}+\frac{\left|\bar{t}-\bar{t}^{\prime}\right|^{2}}{\gamma^{2}} \leq \beta .
$$

Proof By definition, $\left(\bar{t}, \bar{u}, \bar{v}, \bar{t}^{\prime}, \bar{u}^{\prime}, \bar{v}^{\prime}\right)$ satisfies, for all $(t, u, v),\left(t^{\prime}, u^{\prime}, v^{\prime}\right) \in \mathcal{R}$

$$
\begin{aligned}
& Z(\bar{t}, \bar{u}, \bar{v})-V\left(\bar{t}^{\prime}, \bar{u}^{\prime}, \bar{v}^{\prime}\right)-\frac{\left|\bar{u}-\bar{u}^{\prime}\right|^{2}}{\gamma^{2}}-\frac{\left|\bar{v}-\bar{v}^{\prime}\right|^{2}}{\gamma^{2}}-\frac{\left|\bar{t}-\bar{t}^{\prime}\right|^{2}}{\gamma^{2}} \geq \\
& Z(t, u, v)-V\left(t^{\prime}, u^{\prime}, v^{\prime}\right)-\frac{\left|u-u^{\prime}\right|^{2}}{\gamma^{2}}-\frac{\left|v-v^{\prime}\right|^{2}}{\gamma^{2}}-\frac{\left|t-t^{\prime}\right|^{2}}{\gamma^{2}}
\end{aligned}
$$

Let us take $(t, u, v)=\left(t^{\prime}, u^{\prime}, v^{\prime}\right)$, then

$$
\mathbb{M}_{\gamma} \geq Z(t, u, v)-V(t, u, v) .
$$

We then obtain $\mathbb{M} \leq \mathbb{M}_{\gamma}$. Let $r^{2}=\|Z\|_{\mathcal{R}}+\|V\|_{\mathcal{R}}$. We have

$$
0<\mathbb{M} \leq \mathbb{M}_{\gamma} \leq r^{2}-\frac{\left|\bar{u}-\bar{u}^{\prime}\right|^{2}}{\gamma^{2}}-\frac{\left|\bar{v}-\bar{v}^{\prime}\right|^{2}}{\gamma^{2}}-\frac{\left|\bar{t}-\bar{t}^{\prime}\right|^{2}}{\gamma^{2}}
$$

Hence

$$
\left|\bar{u}-\bar{u}^{\prime}\right| \leq \gamma r, \quad\left|\bar{v}-\bar{v}^{\prime}\right| \leq \gamma r \text { and }\left|\bar{t}-\bar{t}^{\prime}\right| \leq \gamma r
$$


We also have that

$$
\mathbb{M} \leq \mathbb{M}_{\gamma}=Z(\bar{t}, \bar{u}, \bar{v})-V\left(\bar{t}^{\prime}, \bar{u}^{\prime}, \bar{v}^{\prime}\right)-\frac{\left|\bar{u}-\bar{u}^{\prime}\right|^{2}}{\gamma^{2}}-\frac{\left|\bar{v}-\bar{v}^{\prime}\right|^{2}}{\gamma^{2}}-\frac{\left|\bar{t}-\bar{t}^{\prime}\right|^{2}}{\gamma^{2}} .
$$

The function $Z$ is upper semi-continuous, then for any $\beta>0$, there exists $\gamma_{0}$, such that for any $\gamma \leq \gamma_{0}$, using (17), we get

$$
\mathbb{M} \leq Z(\bar{t}, \bar{u}, \bar{v})-V\left(\bar{t}^{\prime}, \bar{u}^{\prime}, \bar{v}^{\prime}\right) \leq Z\left(\bar{t}^{\prime}, \bar{u}^{\prime}, \bar{v}^{\prime}\right)-V\left(\bar{t}^{\prime}, \bar{u}^{\prime}, \bar{v}^{\prime}\right)+\beta \leq \mathbb{M}+\beta .
$$

Using (18), we get the result of the lemma.

Let us now prove the theorem.

\section{Proof}

Case $\bar{t}$ and $\bar{t}^{\prime}$ smaller than $T$ We recall that $\left(\bar{t}, \bar{u}, \bar{v}, \bar{t}^{\prime}, \bar{u}^{\prime}, \bar{v}^{\prime}\right)$ satisfies $\forall(t, u, v) \in \mathcal{R}$,

$$
\begin{aligned}
& Z(\bar{t}, \bar{u}, \bar{v})-V\left(\bar{t}^{\prime}, \bar{u}^{\prime}, \bar{v}^{\prime}\right)-\frac{\left|\bar{u}-\bar{u}^{\prime}\right|^{2}}{\gamma^{2}}-\frac{\left|\bar{v}-\bar{v}^{\prime}\right|^{2}}{\gamma^{2}}-\frac{\left|\bar{t}-\bar{t}^{\prime}\right|^{2}}{\gamma^{2}} \geq \\
& Z(t, u, v)-V\left(\bar{t}^{\prime}, \bar{u}^{\prime}, \bar{v}^{\prime}\right)-\frac{\left|u-\bar{u}^{\prime}\right|^{2}}{\gamma^{2}}-\frac{\left|v-\bar{v}^{\prime}\right|^{2}}{\gamma^{2}}-\frac{\left|t-\bar{t}^{\prime}\right|^{2}}{\gamma^{2}}
\end{aligned}
$$

Let

$$
\Phi_{1}(t, u, v)=V\left(\bar{t}^{\prime}, \bar{u}^{\prime}, \bar{v}^{\prime}\right)+\frac{\left|u-\bar{u}^{\prime}\right|^{2}}{\gamma^{2}}+\frac{\left|v-\bar{v}^{\prime}\right|^{2}}{\gamma^{2}}+\frac{\left|t-\bar{t}^{\prime}\right|^{2}}{\gamma^{2}},
$$

$\Phi_{1} \in C^{1}(\mathcal{R})$. This inequality means that $(\bar{t}, \bar{u}, \bar{v})$ is a maximal point of $Z(t, u, v)-\Phi_{1}(t, u, v)$. We also have, $\forall\left(t^{\prime}, u^{\prime}, v^{\prime}\right) \in \mathcal{R}$,

$$
\begin{aligned}
& Z(\bar{t}, \bar{u}, \bar{v})-V\left(\bar{t}^{\prime}, \bar{u}^{\prime}, \bar{v}^{\prime}\right)-\frac{\left|\bar{u}-\bar{u}^{\prime}\right|^{2}}{\gamma^{2}}-\frac{\left|\bar{v}-\bar{v}^{\prime}\right|^{2}}{\gamma^{2}}-\frac{\left|\bar{t}-\bar{t}^{\prime}\right|^{2}}{\gamma^{2}} \geq \\
& Z(\bar{t}, \bar{u}, \bar{v})-V\left(t^{\prime}, u^{\prime}, v^{\prime}\right)-\frac{\left|\bar{u}-u^{\prime}\right|^{2}}{\gamma^{2}}-\frac{\left|\bar{v}-v^{\prime}\right|^{2}}{\gamma^{2}}-\frac{\left|\bar{t}-t^{\prime}\right|^{2}}{\gamma^{2}}
\end{aligned}
$$

Let

$$
\Phi_{2}\left(t^{\prime}, u^{\prime}, v^{\prime}\right)=Z(\bar{t}, \bar{u}, \bar{v})-\frac{\left|\bar{u}-u^{\prime}\right|^{2}}{\gamma^{2}}-\frac{\left|\bar{v}-v^{\prime}\right|^{2}}{\gamma^{2}}-\frac{\left|\bar{t}-t^{\prime}\right|^{2}}{\gamma^{2}} .
$$

$\Phi_{2} \in C^{1}(\mathcal{R})$. This inequality means that $\left(\bar{t}^{\prime}, \bar{u}^{\prime}, \bar{v}^{\prime}\right)$ is a minimal point of $V\left(t^{\prime}, u^{\prime}, v^{\prime}\right)-\Phi_{2}\left(t^{\prime}, u^{\prime}, v^{\prime}\right)$. Then

$$
\begin{aligned}
-\frac{\partial \Phi_{1}}{\partial t}+Z(\bar{t}, \bar{u}, \bar{v})+\min _{\tau \in\left[\tau^{-}, \tau^{+}\right]} \tau\left[-\frac{\partial \Phi_{1}}{\partial u} \bar{u}-\frac{\partial \Phi_{1}}{\partial v} \bar{v}+\mathrm{e}^{\bar{t}} L(\bar{v})\right] & \leq \delta^{\prime}<0, \\
-\frac{\partial \Phi_{1}}{\partial v}-\mathrm{e}^{\bar{t}} C^{+} & \leq \delta<0, \\
\frac{\partial \Phi_{1}}{\partial v}+\mathrm{e}^{\bar{t}} C^{-} & \leq \delta<0 .
\end{aligned}
$$

These inequalities can be written as

$$
\begin{aligned}
& \frac{-2\left(\bar{t}-\bar{t}^{\prime}\right)}{\gamma^{2}}+Z(\bar{t}, \bar{u}, \bar{v})+ \\
& \min _{\tau \in\left[\tau^{-}, \tau^{+}\right]} \tau\left[-\frac{2\left(\bar{u}-\bar{u}^{\prime}\right)}{\gamma^{2}} \bar{u}-\frac{2\left(\bar{v}-\bar{v}^{\prime}\right)}{\gamma^{2}} \bar{v}+\mathrm{e}^{\bar{t}} L(\bar{v})\right] \leq \delta^{\prime}<0,
\end{aligned}
$$




$$
\begin{gathered}
-\frac{2\left(\bar{v}-\bar{v}^{\prime}\right)}{\gamma^{2}}-\mathrm{e}^{\bar{t}} C^{+} \leq \delta<0, \\
\frac{2\left(\bar{v}-\bar{v}^{\prime}\right)}{\gamma^{2}}+\mathrm{e}^{\bar{t}} C^{-} \leq \delta<0 .
\end{gathered}
$$

We also have

$$
\begin{aligned}
& \max \left\{-\frac{2\left(\bar{t}-\bar{t}^{\prime}\right)}{\gamma^{2}}+V\left(\bar{t}^{\prime}, \bar{u}^{\prime}, \bar{v}^{\prime}\right)+\right. \\
& \min _{\tau \in\left[\tau^{-}, \tau^{+}\right]} \tau\left[-\frac{2\left(\bar{u}-\bar{u}^{\prime}\right)}{\gamma^{2}} \bar{u}^{\prime}-\frac{2\left(\bar{v}-\bar{v}^{\prime}\right)}{\gamma^{2}} \bar{v}^{\prime}+\mathrm{e}^{\bar{t}^{\prime}} L\left(\bar{v}^{\prime}\right)\right], \\
& \left.\frac{-2\left(\bar{v}-\bar{v}^{\prime}\right)}{\gamma^{2}}-\mathrm{e}^{\bar{t}^{\prime}} C^{+}, \frac{2\left(\bar{v}-\bar{v}^{\prime}\right)}{\gamma^{2}}+\mathrm{e}^{\bar{t}^{\prime}} C^{-}\right\} \geq 0 .
\end{aligned}
$$

Let $\beta=1$ in Lemma 3.2 and use the fact that $\exp$ function is uniformly continuous over $[0, T]$, then one can find $0<\gamma_{1} \leq \gamma_{0}$ such that for all $\gamma \leq \gamma_{1}$, we have $\left|\mathrm{e}^{\bar{t}}-\mathrm{e}^{\bar{t}^{\prime}}\right|<-\delta / 2 C^{+}$, and likewise, $0<\gamma_{2} \leq \gamma_{0}$ such that for all $\gamma \leq \gamma_{2}$, we have $\left|\mathrm{e}^{\bar{t}^{\prime}}-\mathrm{e}^{\bar{t}}\right|<\delta / 2 C^{-}$. Using (21) and (22), we get, for all $\gamma \leq \min \left(\gamma_{1}, \gamma_{2}\right)$,

$$
\begin{gathered}
-\frac{2\left(\bar{v}-\bar{v}^{\prime}\right)}{\gamma^{2}}-\mathrm{e}^{\bar{t}^{\prime}} C^{+} \leq \frac{\delta}{2}<0, \\
\frac{2\left(\bar{v}-\bar{v}^{\prime}\right)}{\gamma^{2}}+\mathrm{e}^{\bar{t}^{\prime}} C^{-} \leq \frac{\delta}{2}<0 .
\end{gathered}
$$

We then get

$$
\begin{aligned}
& \frac{-2\left(\bar{t}-\bar{t}^{\prime}\right)}{\gamma^{2}}+V\left(\bar{t}^{\prime}, \bar{u}^{\prime}, \bar{v}^{\prime}\right)+ \\
& \min _{\tau \in\left[\tau^{-}, \tau^{+}\right]} \tau\left[-\frac{2\left(\bar{u}-\bar{u}^{\prime}\right)}{\gamma^{2}} \bar{u}^{\prime}-\frac{2\left(\bar{v}-\bar{v}^{\prime}\right)}{\gamma^{2}} \bar{v}^{\prime}+\mathrm{e}^{\bar{t}^{\prime}} L\left(\bar{v}^{\prime}\right)\right] \geq 0 .
\end{aligned}
$$

We subtract (23) to (20) and we get

$$
\begin{aligned}
& Z(\bar{t}, \bar{u}, \bar{v})-V\left(\bar{t}^{\prime}, \bar{u}^{\prime}, \bar{v}^{\prime}\right) \leq \\
& \max _{\tau \in\left[\tau^{-}, \tau^{+}\right]} \tau\left[\frac{2\left(\bar{u}-\bar{u}^{\prime}\right)^{2}}{\gamma^{2}}+\frac{2\left(\bar{v}-\bar{v}^{\prime}\right)^{2}}{\gamma^{2}}+\mathrm{e}^{\bar{t}^{\prime}} L\left(\bar{v}^{\prime}\right)-\mathrm{e}^{\bar{t}} L(\bar{v})\right] \leq \\
& \max _{\tau \in\left[\tau^{-}, \tau^{+}\right]} \tau\left[\frac{2\left(\bar{u}-\bar{u}^{\prime}\right)^{2}}{\gamma^{2}}+\frac{2\left(\bar{v}-\bar{v}^{\prime}\right)^{2}}{\gamma^{2}}+\mathrm{e}^{\bar{t}^{\prime}}\left(L\left(\bar{v}^{\prime}\right)-L(\bar{v})\right)+\left(\mathrm{e}^{\bar{t}^{\prime}}-\mathrm{e}^{\bar{t}}\right) L(\bar{v})\right] .
\end{aligned}
$$

We use again as previously Lemma 3.2 with $\beta=1$ and the fact that $L$ and the exp function are uniformly continuous to get, for all $\varepsilon>0$, one can find $0<\gamma_{3} \leq \min \left(\gamma_{1}, \gamma_{2}\right)$ and $0<\gamma_{4} \leq$ $\min \left(\gamma_{1}, \gamma_{2}\right)$ such that for all $\gamma \leq \min \left(\gamma_{3}, \gamma_{4}\right)$, we have $\left|L\left(\bar{v}^{\prime}\right)-L(\bar{v})\right| \leq \varepsilon$ and $\left|\mathrm{e}^{\bar{t}^{\prime}}-\mathrm{e}^{\bar{t}}\right|<\varepsilon$. Finally, we use Lemma 3.2 with arbitrary $\beta$ to find $0<\gamma_{5} \leq \min \left(\gamma_{3}, \gamma_{4}\right)$ such that for all $\gamma \leq \gamma_{5}$, we have $2\left(\bar{u}-\bar{u}^{\prime}\right)^{2} / \gamma^{2}+2\left(\bar{v}-\bar{v}^{\prime}\right)^{2} / \gamma^{2} \leq 2 \beta$. Then, for all $\gamma \leq \gamma_{5}$, we get

$$
Z(\bar{t}, \bar{u}, \bar{v})-V\left(\bar{t}^{\prime}, \bar{u}^{\prime}, \bar{v}^{\prime}\right) \leq 2 \beta \tau^{*}+\varepsilon \tau^{*}\left(\mathrm{e}^{T}+\|L\|_{\infty}\right) .
$$

As $\beta$ and $\varepsilon$ are arbitrary, we get $Z(\bar{t}, \bar{u}, \bar{v})-V\left(\bar{t}^{\prime}, \bar{u}^{\prime}, \bar{v}^{\prime}\right) \leq 0$. Using (19), we get $\mathbb{M} \leq 0$ and then $Z(t, u, v) \leq V(t, u, v), \forall(t, u, v) \in[0, T) \times \mathbb{R}^{2}$. We let $\alpha \rightarrow 0$ and $\mu \rightarrow 1$ to get $U(t, u, v) \leq$ $V(t, u, v)$ for all $\forall(t, u, v) \in[0, T) \times \mathbb{R}^{2}$. 
Case $\bar{t}$ or $\bar{t}^{\prime}$ equal to $T$ In the case where $\bar{t}$ or $\bar{t}^{\prime}$ are equal to $T$, QVI (13) cannot be used, because it assumes $t<T$. Because of the fact that $U$ is a viscosity subsolution of (13) and $V$ is a viscosity supersolution of (13), we have the following inequality

$$
\forall(u, v) \in \mathbb{R}^{2}, \quad U(T, u, v) \leq \mathrm{e}^{T} N(u, v) \leq V(T, u, v) .
$$

We then get the result of the theorem.

Theorem 3.2 The quasi-variational inequality (13) has a unique bounded continuous viscosity solution.

Proof Existence follows from propositions 2.2 and 3.1. Now, assume that it has two bounded continuous viscosity solutions $\Gamma_{1}$ and $\Gamma_{2}$. We first use $\Gamma_{1}$ as a bounded upper semi-continuous subsolution, and $\Gamma_{2}$ as a bounded viscosity supersolution, and we use the result of Theorem 3.1. We change the role of $\Gamma_{1}$ and $\Gamma_{2}$ and then we get $\Gamma_{1}(t, u, v)=\Gamma_{2}(t, u, v)$ for all $(t, u, v) \in[0, T] \times \mathbb{R}^{2}$.

\section{Corollary 3.1}

1. Theorem 2.2 is proved.

2. The Value function of the continuous trading problem is the unique viscosity solution of (8).

\section{Proof}

1. Theorem 2.2 is a direct consequence of theorem 3.2 and of proposition 3.1.

2. The DQVI (8) has a viscosity solution by theorem 2.1. It is unique, because if it had two solutions $W_{1}$ and $W_{2}$, with $(t, u, v)$ such that $W_{1}(t, u, v) \neq W_{2}(t, u, v)$, by taking $R$ large enough in the modified game, we could manage to get $(u, v) \in \mathcal{R}$, and therefore a contradiction of theorem 2.2.

As mentioned at the beginning of paragraph 2.2.2, as a consequence, the other results in [8] that depend on this uniqueness are proved, most noticeably convergence of the Value function of the discrete trading problem to that of the continuous trading one, and therefore convergence of the fast algorithm toward the Value function sought.

Acknowledgment The uniqueness proof owes much to discussions with Guy Barles, of University François Rabelais, Tours, France.

\section{References}

[1] Bardi, M., Capuzzo-Dolcetta, I.: Optimal Control and Viscosity Solutions of Hamilton-JacobiBellman Equations. Birkhaüser, Basel (1997)

[2] Bardi M, Falcone M, Soravia P.: Numerical methods for pursuit-evasion games via viscosity solutions. Stochastic and Differential Games, Annals of the International Society of Dynamic Games, vol. 4, Bardi M, Raghavan T, Parthasarathy T (eds.). Birkhaüser, 105-175 (1999)

[3] Bensoussan, A., Lions, JL.: Contrôle impulsionnel et inéquations quasi-variationnelles. Dunod, Paris (1982) 
[4] Bernhard, P.: Une approche déterministe de l'évaluation d'options. Optimal Control and Partial Differential Equations, vol. in honor of Professor Alain Bensoussan's 60th birthday. Menaldi JL, Rofman E, Sulem A (eds.), 511-520. IOS Press, Amsterdam (2001)

[5] Bernhard, P.: The robust control approach to option pricing and interval models: an overview. Numerical Methods in Finance. Breton M, Ben-Ameur H (eds.), 91-108. Springer, New York (2005)

[6] Bernhard, P.: Singular surfaces in differential games, an introduction. Differential games and Applications, Lecture Notes in Information and Control Sciences. Haggedorn P, Olsder G, Knobloch H (eds.), vol. 3, 1-33. Springer Verlag, Berlin (1977)

[7] Bernhard, P., El Farouq, N.: A robust control approach to option pricing: the uniqueness theorem. EPSRC Symposium Workshop on game theory for finance, social and biological sciences, University of Warwick, UK (2010), and in: Game Theory and Applications, vol. 16, 1-18. Nova Publishers, New York (2013)

[8] Bernhard, P., El Farouq, N., Thiery, S.: Robust control approach to option pricing: representation theorem and fast algorithm. SIAM J. Control Optim. 46, 2280-2302 (2007)

[9] Bernhard, P., El Farouq, N., Thiery, S.: An impulsive differential game arising in finance with interesting singularities. In: Annals of the ISDG, vol. 8, 335-363. Birkhaüser, Basel (2006)

[10] Bernhard, P., Engwerda, J., Roorda, B., Schumacher, H., Kolokoltsov, V., Aubin, JP., SaintPierre, P.: The Interval Market Model in Mathematical Finance: a Game Theoretic Approach. Springer, New York (2012)

[11] El Farouq, N.: Degenerate first-order quasi-variational inequalities: an approach to approximate the value function. Preprint

[12] El Farouq, N., Barles, G., Bernhard, P.: Deterministic Minimax Impulse Control. Applied Mathematics and Optimization. 46, pp. 353-378 (2010)

[13] Evans LC, Souganidis PE. Differential games and representation formulas for solutions of Hamilton-Jacobi-Isaacs equations. Indiana University Mathematical Journal 33, 773-797 (1984)

[14] Souganidis PE. Two-player, zero-sum differential games and viscosity solutions. Stochastic and Differential Games, Annals of the International Society of Dynamic Games, vol. 4, Bardi M, Raghavan T, Parthasarathy T (eds.). Birkhaüser, 69-104 (1999) 